\title{
Wallerian degeneration of the pyramidal tract after a thrombotic stroke
}
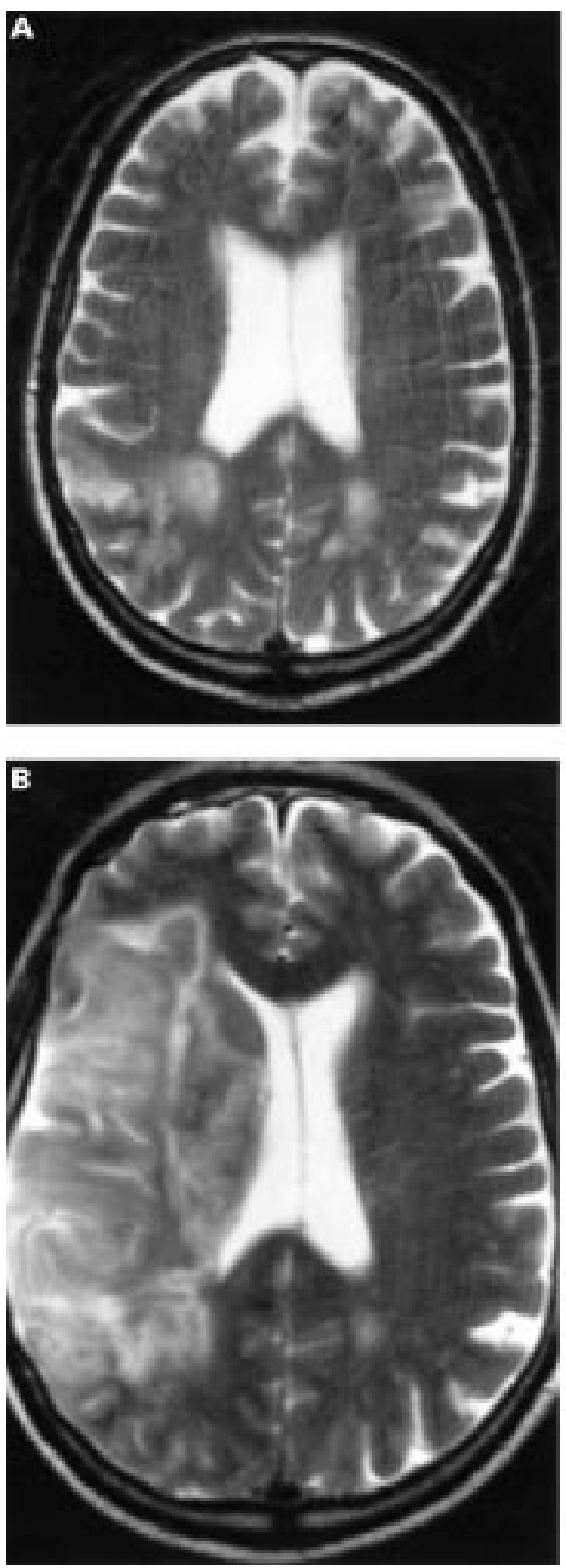

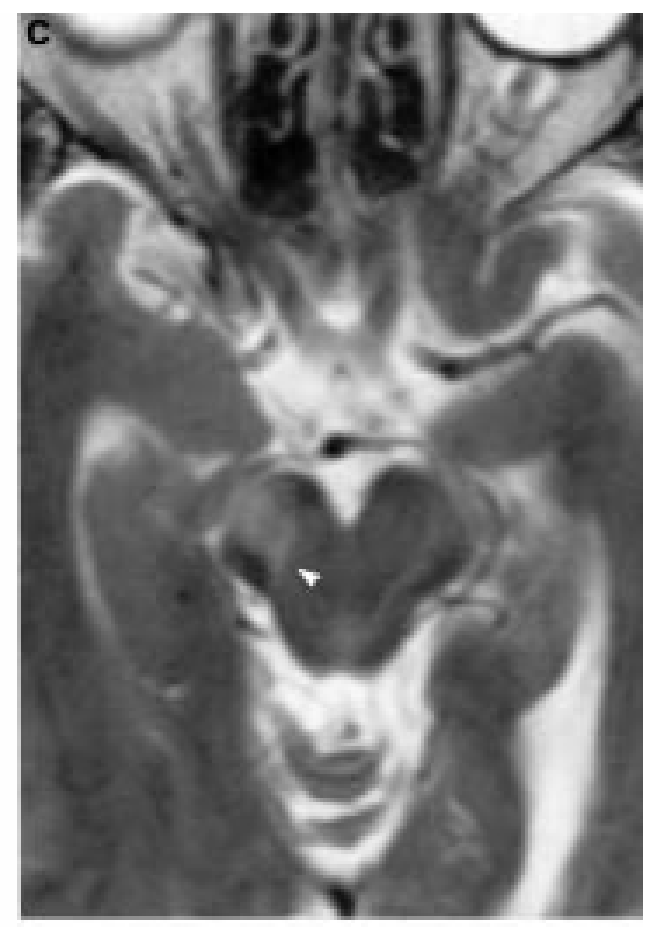

A sixty two year old non-insulin dependent diabetic patient was admitted with evolving weakness in the left upper limb of one day's duration.

Examination disclosed mild weakness of grip on the left side and an extensor left plantar response. Brain MRI performed two days after admission showed a borderzone infarct in the right cerebral hemisphere (figure A). The intracranial portion of the carotid and basilar arteries were patent containing normal signal voids. One week after the MRI he deteriorated and developed a complete left hemiplegia with sensory inattention. MR I undertaken at this stage showed a large right middle cerebral artery territory infarct (figure B) with loss of the signal void from the right carotid artery. The mid-portion of the right cerebral peduncle contained a well defined band of altered signal in the region of the pyramidal tract (figure C).

Various mechanisms have been suggested for the changes in MR signal from the pyramidal tract after a cortical ischaemic or haemorrhagic event. These include increased water content, glial proliferation, and compartmentalisation of the extracellular and intracellular water protons. ${ }^{1}$

These signal changes usually take about four weeks to develop after cortical ischaemia and their early identification in this case is unusual.

D G RAO P R LYONS

Royal United Hospital, Combe Park, Bath BA1 3NG, UK 